Part 2. Survey Projects 


\title{
WIDE-FIELD LOW-FREQUENCY IMAGING WITH THE VLA
}

\author{
N.E. KASSIM, D.S. BRIGGS AND R.S. FOSTER \\ Naval Research Laboratory, Washington
}

\section{Introduction}

The $330 \mathrm{MHz}$ observing system at the VLA is a potentially powerful survey system. It can map fields many degrees in size quickly and at high sensitivity. However imaging characteristics unique to this data pose excessive computational burdens on conventional mapping systems. Hence while data acquisition is quick and efficient, data reduction has been difficult and slow. Here we describe how powerful new scalable processing algorithms have been used to generate the first full resolution $330 \mathrm{MHz}$ images, demonstrating also that lower resolution survey work is now a tractable problem.

\section{Solution to The Wide-Field Imaging Problem: DRAGON}

Imaging at low frequencies is complicated because the VLA is a noncoplanar array (Cornwell and Perly 1992). At commonly used centimeter wavelengths, 2-D approximations to the celestial sphere work well. However for the large fields of view and enhanced source count densities encountered at $330 \mathrm{MHz}$ these approximations break down and introduce phase errors which severely limit sensitivity. Tim Cornwell of the NRAO has implemented a full solution to this problem via the polyhedron algorithm DRAGON. We have recently ported DRAGON to the SGI Power Challenge Array at the Army Research Laboratory.

\subsection{LOW RESOLUTION IMAGES REQUIRE CONTIGUOUS FACETING}

Low resolution VLA data acquired in the $\mathrm{B}(<12 \mathrm{~km}), \mathrm{C}(<3 \mathrm{~km})$ and D $(<1 \mathrm{~km})$ configurations can be handled by contiguous faceting. Here the 3 -D image volume is broken up into a set of contiguous 2-D facets. The 
advantage over a full 3-D transform is a significant savings in computational expense. Images produced in this way are now achieving thermal and confusion limited sensitives on a routine basis. See Frail, Kassim, and Weiler (1994) and Kassim \& Frail (1996) for examples. Common "B array DRAGONs" take several days to run on conventional workstations but only a few hours on the Power Challenge Array.

\subsection{HIGH RESOLUTION IMAGING WITH TARGETED FACETING.}

Full pixellation of the primary beam in the A array $(<36 \mathrm{~km})$ is impossible even with the most powerful contemporary machines. Hence we have implemented Cornwell's suggested technique of targeted faceting in which small outlier fields are placed only on the hundreds of small-diameter background sources which must be deconvolved, avoiding pixellation of large regions of empty sky. A smaller contiguously faceted region is centered on the target source. The location of the outlier fields is determined from a tapered resolution image or an image from a smaller VLA configuration. This technique has been demonstrated on the supernova remnant $\mathrm{W} 49 \mathrm{~B}$, producing the first full resolution $330 \mathrm{MHz}$ image from the VLA generated using proper 3-D algorithms. This image would have taken months to produce on conventional workstations but was produced in only a few days on the Power Challenge Array.

\section{Summary}

We have implemented algorithms to achieve thermal noise limited sensitivity, wide-field $330 \mathrm{MHz}$ images at the full resolution of the VLA. We have successfully implemented this code on scaled processing computers and generated images in manageable lengths of time. This capability allows data reduction to keep up with data acquisition which could be quick and efficient for surveying purposes. Hence the capability of undertaking sensitive, high resolution large sky surveys with this system is realized. As a look forward we estimate that only $\sim 40$ hours of telescope time would be required to survey the first quadrant of the Galactic plane with a latitude coverage of $\pm 3^{\circ}$ at $1 \mathrm{mJy}$ sensitivity. Science goals would define the required resolution.

\section{References}

Cornwell, T.J., and Perley, R.A., 1992, Astron.Astrophys. 261, 353. Frail, D.A., Kassim, N.E. and Weiler, K.W.,1994, Astron.J. 107, 1120. Kassim. N.E. and Frail, D.A., 1996, Mon.Not.R.astron.Soc. in press 